\title{
Cost-effective Tapering Algorithm in Patients with Rheumatoid Arthritis: Combination of Multibiomarker Disease Activity Score and Autoantibody Status
}

Melanie Hagen, Matthias Englbrecht, Judith Haschka, Michaela Reiser, Arnd Kleyer, Axel Hueber, Bernhard Manger, Camille Figueiredo, Jayme Fogagnolo Cobra, Hans-Peter Tony, Stephanie Finzel, Stefan Kleinert, Jörg Wendler, Florian Schuch, Monika Ronneberger, Martin Feuchtenberger, Martin Fleck, Karin Manger, Wolfgang Ochs, Hans-Martin Lorenz, Hubert Nüsslein, Rieke Alten (D), Jörg Henes, Klaus Krüger, Georg Schett, and Jürgen Rech

\begin{abstract}
Objective. To analyze the effect of a risk-stratified disease-modifying antirheumatic drug (DMARD)tapering algorithm based on multibiomarker disease activity (MBDA) score and anticitrullinated protein antibodies (ACPA) on direct treatment costs for patients with rheumatoid arthritis (RA) in sustained remission.

Methods. The study was a posthoc retrospective analysis of direct treatment costs for 146 patients with RA in sustained remission tapering and stopping DMARD treatment, in the prospective randomized RETRO study. MBDA scores and ACPA status were determined in baseline samples of patients continuing DMARD (arm 1), tapering their dose by 50\% (arm 2), or stopping after tapering (arm 3). Patients were followed over 1 year, and direct treatment costs were evaluated every 3 months. MBDA and ACPA status were used as predictors creating a risk-stratified tapering algorithm based on relapse rates.

Results. RA patients with a low MBDA score (<30 units) and negative ACPA showed the lowest relapse risk (19\%), while double-positive patients showed high relapse risk (61\%). In ACPA-negative and MBDA-negative (<30 units), and ACPA or MBDA single-positive ( $>30$ units) groups, DMARD tapering appears feasible. Considering only patients without flare, direct costs for synthetic and biologic DMARD in the ACPA/MBDA-negative and single positive groups $(n=41)$ would have been $€ 372,245.16$ for full-dose treatment over 1 year. Tapering and stopping DMARD in this low-risk relapse group allowed a reduction of $€ 219,712.03$ of DMARD costs. Average reduction of DMARD costs per patient was $€ 5358.83$.

Conclusion. Combining MBDA score and ACPA status at baseline may allow risk stratification for successful DMARD tapering and cost-effective use of biologic DMARD in patients in deep remission as defined by the 28-joint count Disease Activity Score using erythrocyte sedimentation rate. (First Release December 1 2018; J Rheumatol 2019;46:460-6; doi:10.3899/jrheum.180028)
\end{abstract}

Key Indexing Terms:

RHEUMATOID ARTHRITIS DRUG COSTS MULTIBIOMARKER DISEASE ACTIVITY TAPERING REMISSION ANTICYCLIC CITRULLINATED PROTEIN ANTIBODIES

\begin{abstract}
From the Friedrich-Alexander-University Erlangen-Nuremberg (FAU), Department of Internal Medicine 3 - Rheumatology and Immunology, Universitätsklinikum Erlangen, Erlangen, Germany; St. Vincent Hospital, Vinforce Study Group, Medical University of Vienna, Vienna, Austria; Institutio de Rheumatologia, São Paolo, Brazil; University of Würzburg, Internal Medicine 2, Würzburg; University Medical Center Freiburg, Rheumatology and Clinical Immunology, Freiburg; Rheumatology Clinical Practice Erlangen, Erlangen; Rheumatology Practice and Department of Internal Medicine 2, Clinic Burghausen, Burghausen; Asklepios Medical Center, Department of Rheumatology and clinical Immunology, Bad Abbach; Rheumatology Practice Bamberg, Bamberg; Rheumatology Practice Bayreuth, Bayreuth; University of Heidelberg, Medicine 5, Heidelberg; Rheumatology Practice Nuremberg, Nuremberg; Schlosspark Klinik, Internal Medicine/Rheumatology, Berlin; University of Tübingen, Centre for Interdisciplinary Clinical Immunology, Tübingen;

Praxiszentrum St. Bonifatius, Munich, Germany.

M. Hagen, BSc, CandMed, FAU, Department of Internal Medicine 3 Rheumatology and Immunology, Universitätsklinikum Erlangen;
\end{abstract}

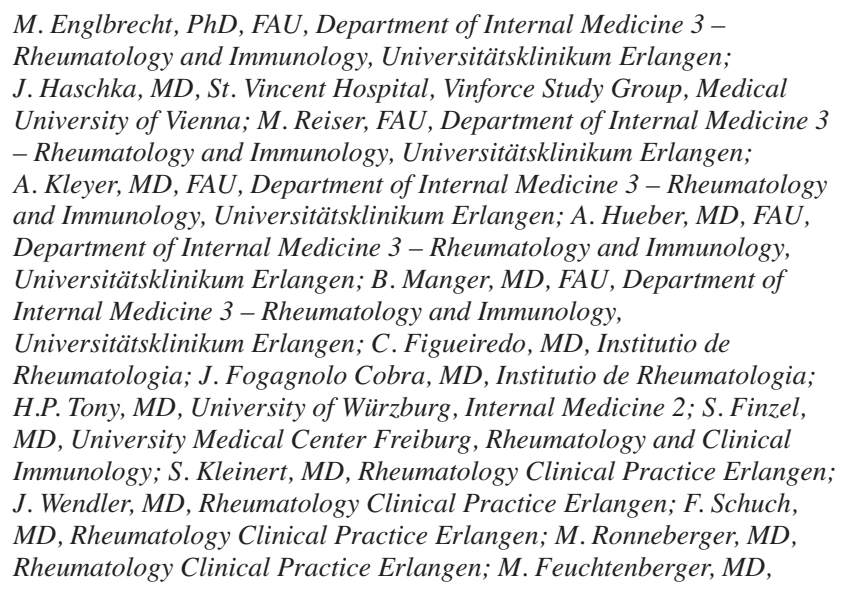


Rheumatoid arthritis (RA) is a prototype inflammatory disease with a prevalence of up to $1 \%$ worldwide. RA is characterized by chronic joint inflammation requiring longterm treatment ${ }^{1}$. Disease-modifying antirheumatic drug (DMARD) treatment of RA, though often highly effective, is associated with substantial healthcare costs and possible side effects related to longterm treatment. With the continuous improvement of RA therapy and treat-to-target strategies, the number of patients achieving a symptom-free state (remission) is steadily increasing, suggesting the need for stratified approaches for tapering and stopping DMARD treatment in patients with low risk of relapse $\mathrm{e}^{2,3}$.

The concept of tapering and stopping DMARD in RA patients in sustained remission has been discussed in a substantial number of clinical studies ${ }^{4}$. They indicated that in cases of stable clinical remission for more than 6 months, tapering and stopping of DMARD is feasible only in a subset of patients with RA. Quality of remission [e.g., deep remission such as Boolean, vs more "shallow" remission such as in the 28-joint count Disease Activity Score using erythrocyte sedimentation rate (DAS28-ESR)] may play a role in deciding whether a patient with RA experiences relapse. The key question is to define those patients in remission who can successfully taper and stop DMARD treatment. Such predictive modeling of successful versus unsuccessful DMARD tapering in remission would likely allow the prevention of overtreatment of RA patients, with the consequences of reducing side effects and drug costs ${ }^{5}$.

In previous data from the RETRO study, a randomized prospective strategy study of DMARD tapering in RA patients in stable DAS28-ESR remission $(<2.6)$, we were able to show that the presence of autoimmunity [anticitrullinated protein antibody (ACPA) positivity] as well as elevation of serum biomarkers of inflammation [multibiomarker disease activity (MBDA)] were independent predictors for relapse, if DMARD were tapered and stopped $^{6,7}$. The combination of ACPA status and MBDA has shown to be a possible feasible approach for defining prediction models of relapse risk in patients with RA in remission who are undergoing DMARD tapering ${ }^{7}$.

Rheumatology Practice and Department of Internal Medicine 2, Clinic Burghausen; M. Fleck, MD, Asklepios Medical Center, Department of Rheumatology and clinical Immunology; K. Manger, MD, Rheumatology Practice Bamberg; W. Ochs, MD, Rheumatology Practice Bayreuth; H.M. Lorenz, MD, University of Heidelberg, Medicine 5; H. Nüsslein, MD, Rheumatology Practice Nuremberg; R. Alten, MD, Schlosspark Klinik, Internal Medicine/Rheumatology; J. Henes, MD, University of Tübingen, Centre for Interdisciplinary Clinical Immunology; K. Krüger, MD,

Praxiszentrum St. Bonifatius; G. Schett, MD, FAU, Department of Internal

Medicine 3 - Rheumatology and Immunology, Universitätsklinikum

Erlangen; J. Rech, MD, FAU, Department of Internal Medicine 3 Rheumatology and Immunology, Universitätsklinikum Erlangen.

Address correspondence to Dr. J. Rech, Friedrich-Alexander-University Erlangen-Nuremberg, Department of Internal Medicine 3 - Rheumatology and Immunology, Universitätsklinikum Erlangen, Ulmenweg 18, 91054 Erlangen, Germany.E-mail: juergen.rech@uk-erlangen.de

Accepted for publication September 7, 2018.
The aim of the present posthoc retrospective analysis of the prospective RETRO study was to show that tapering and stopping DMARD in RA patients in sustained clinical remission is feasible and cost-effective, especially in patients with low risk of relapse based on ACPA negativity and low MBDA status.

\section{MATERIALS AND METHODS}

Patients and inclusion criteria. RETRO is a phase III, prospective, multicenter, open, randomized, controlled, parallel-group study (EudraCT number 2009-015740-42; Figure 1) ${ }^{6}$. The primary objective of the RETRO trial was to evaluate the risk of having a relapse of RA despite tapering or stopping treatment in RA patients in sustained remission. Patients included had to fulfill the American College of Rheumatology (ACR)/European League Against Rheumatism (EULAR) 2010 classification criteria for RA ${ }^{8}$. Prior to study inclusion, patients had to be diagnosed with RA for at least 12 months. Further, patients had to be in stable clinical remission (DAS28-ESR $<2.6)^{9}$ and taking stable doses of conventional (cDMARD) and biological DMARD (bDMARD) for at least 6 months. The present study was approved by the ethics committee of the Friedrich-Alexander-University of Erlangen-Nuremberg, Germany (approval number Az:01_2010) and all local ethics committees of the external centers as well as the Paul-Ehrlich Institute; the study was conducted according to the ethical principles of the Declaration of Helsinki. Patients' written informed consent was obtained to publish the data of the study.

Treatment and followup. Patients fulfilling inclusion criteria were randomized into 3 different arms and observed for 1 year. In arm 1 (control), all DMARD treatments remained unchanged. In arm 2 (tapering), all DMARD treatments were reduced by $50 \%$. In arm 3 (tapering and stopping), all DMARD treatments were reduced by $50 \%$ for the first 6 months and then stopped for a further 6 months. Detailed mode of tapering of the individual drugs has been described elsewhere ${ }^{6}$. Primary efficacy variable was disease activity, as measured by DAS28 using ESR, which was assessed at baseline and after 3,6,9, and 12 months. Relapse was defined as DAS28-ESR $\geq 2.6$. Further details on study procedures and collected demographics as well as disease-related variables are shown elsewhere ${ }^{6}$.

Serum analyses. ACPA status was assessed in baseline serum samples of 146 RETRO patients. ACPA were measured by a commercial anticyclic citrullinated peptide 2 antibody test based on nephelometry (Beckmann Coulter). Cutoff value was $7 \mathrm{IU} / \mathrm{ml}$. MBDA was assessed by commercial Vectra DA test (Crescendo Biosciences). Vectra DA includes 12 inflammation markers: epidermal growth factor, vascular endothelial growth factor A, interleukin 6, serum amyloid A, C-reactive protein (CRP), vascular cell adhesion molecule 1, matrix metalloproteinase 1 (MMP-1), MMP-3, tumor necrosis factor (TNF) receptor 1, human cartilage glycoprotein 39, leptin, and resistin. Detailed measurement is described elsewhere ${ }^{10}$. Based on the serum levels of these markers, a score (MBDA) is calculated as described previously ${ }^{10}$. The MBDA cutoff value for low inflammatory disease activity is 30 units; 30-44 units are defined as moderate disease activity and over 44 units means high disease activity. MBDA scores have been used to measure disease activity in RA $11,12,13,14$.

Treatment cost calculation. At every study visit (baseline, months 3, 6, 9, and 12), direct treatment costs were calculated for each patient. Calculations included cDMARD, bDMARD, and corticosteroids. Other medication was not taken into consideration. Also, indirect costs (e.g., consultation, hospitalization, physiotherapy) were not considered. Drug costs (in euros) were based on the German drug directory and provide real-life values for each patient. Further distinctive features such as contracts with health insurance companies were not taken into account. Costs for the MBDA and ACPA testing were included in baseline costs.

Statistical analysis and risk stratification algorithm. We performed an interim analysis of patients who completed the 12-month study period. With

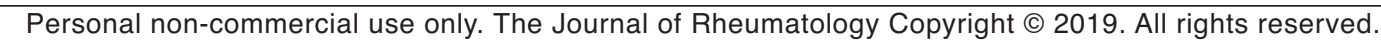




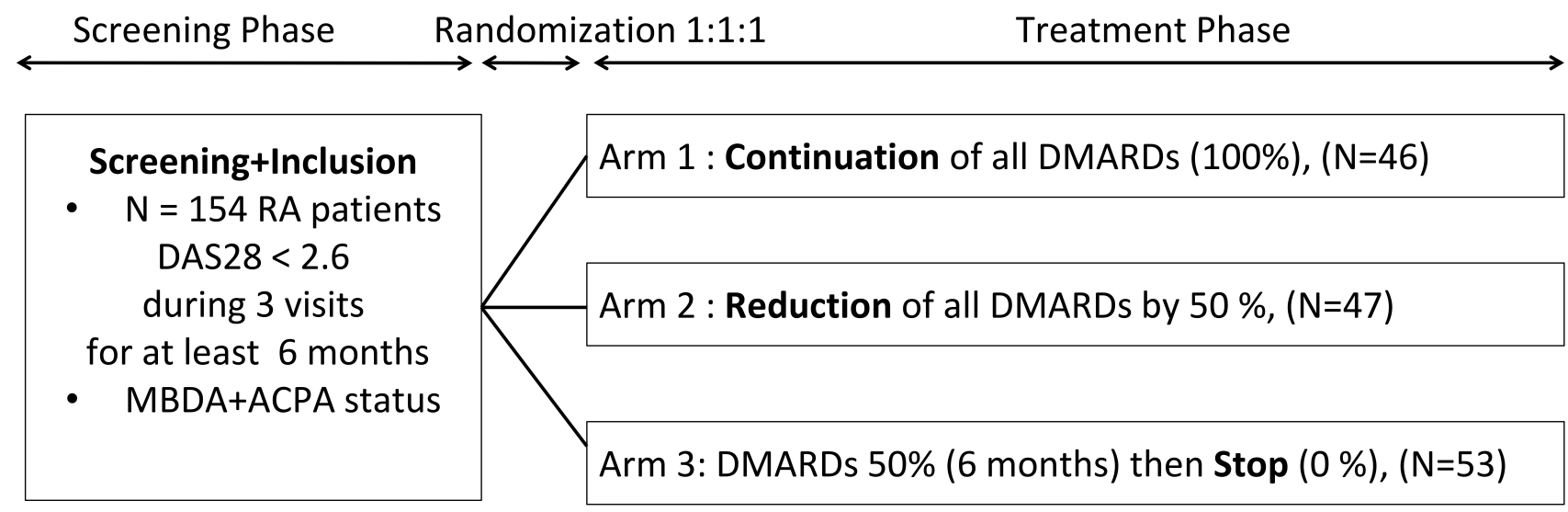

\begin{tabular}{|c|c|c|c|c|c|c|}
\hline-6 & -3 & 0 & 3 & 6 & 9 & 12 \\
\hline & & $\begin{array}{c}\text { DMARD } \\
\text { Costs }\end{array}$ & $\begin{array}{c}\text { DMARD } \\
\text { Costs }\end{array}$ & $\begin{array}{c}\text { DMARD } \\
\text { Costs }\end{array}$ & $\begin{array}{c}\text { DMARD } \\
\text { Costs }\end{array}$ & $\begin{array}{c}\text { DMARD } \\
\text { Costs }\end{array}$ \\
\hline
\end{tabular}

Figure 1. RETRO study design. Prospective randomized controlled trial with 3 treatment arms and 146 patients enrolled. Patients showed DAS28-ESR of $<2.6$ for more than 6 months and were randomized into the 3 study arms (continuation, tapering, stop). ACPA and MBDA status were measured at baseline. Treatment costs were calculated with every study visit every 3 months. Patients were followed over 1 year. RA: rheumatoid arthritis; DMARD: disease-modifying antirheumatic drugs; DAS28-ESR: 28-joint count Disease Activity Score using erythrocyte sedimentation rate; ACPA: anticitrullinated protein antibodies; MBDA: multibiomarker disease activity.

MBDA scores and ACPA previously having been shown to predict relapse of $\mathrm{RA}^{7}$, we designed risk diagrams for RETRO study patients for disease relapse according to the following previously identified predictors: ACPA status (positive/negative), MBDA score (moderate to high/low), and study arm (control, tapering, stopping). Models were performed with stratification for ACPA status only (Model 1) as well as ACPA status combined with MBDA results (Model 2). For both models, cost reductions associated with successful tapering or stopping of DMARD were calculated. Patients with a relapse risk of more than $50 \%$ were excluded from cost calculation because a tapering approach in clinical practice is not feasible. Inferential comparisons of subgroups were calculated using the Kruskal-Wallis test for numerical variables and exact chi-square tests for nominal characteristics. IBM SPSS version 21 was used for the analyses, and $p$ values $\leq 0.05$ were considered statistically significant. All results are presented in mean \pm SD if not stated otherwise.

\section{RESULTS}

Baseline characteristics. One-year followup data of 146 patients enrolled in the RETRO study were available. The patients were randomized 1:1:1 in the 3 different treatment arms: arm 1 (control, $n=46$ ), arm 2 (tapering, $n=47$ ), arm 3 (tapering and stopping, $n=53$; Figure 1). Table 1 shows the baseline characteristics of patients. Mean age was 56.1 $( \pm 1.061)$ years, mean disease duration $7.1( \pm 0.588)$ years, and $56.8 \%(\mathrm{n}=83)$ were female. All patients were in sustained clinical remission with a mean DAS28-ESR score of $1.71( \pm 0.056)$ at baseline. There were $79.5 \%$ of patients taking cDMARD treatment with methotrexate $(n=116)$, while $39.0 \%(n=57)$ of patients were taking bDMARD, including TNF inhibitors (tocilizumab and abatacept). ACPA positivity was found in $56.2 \%(\mathrm{n}=82)$ and MBDA scores over 30 units in $43.2 \%$ of the patients $(n=63)$. The distribution of MBDA positivity differed significantly $(\mathrm{p}=0.018)$ between the 3 treatment arms. Baseline characteristics were comparable in the 3 arms.

Risk stratification algorithms using MBDA and ACPA status. Figure 2 shows a risk diagram with only ACPA status as relapse predictor (Model 1). Relapse risk was moderate in ACPA-negative patients when tapering (30\%) or stopping (41.7\%) DMARD treatment. For ACPA-positive patients, relapse risk was higher when tapering $(48.1 \%)$ or stopping (65.52\%) DMARD treatment. Figure 3 shows a risk diagram in which ACPA status was used in conjunction with MBDA score as relapse predictor (Model 2). Relapse risk for patients with double negativity (ACPA-, MBDA < 30) was $33.3 \%$ in patients who tapered treatment and even lower in patients who subsequently stopped treatment $(11 \%)$. With either single positivity for ACPA or MBDA, relapse risk increased with little difference among ACPA and MBDA single positives. Patients with double positivity showed the highest risk for relapse, with $75 \%$ of patients in the tapering group and $81.2 \%$ of patients who tapered and subsequently stopped treatment.

Treatment costs. We defined patients who did not flare as successfully tapered and calculated their saved costs for cDMARD and bDMARD. Table 2A and Table 2B show the 
Table 1. Baseline characteristics.

\begin{tabular}{|c|c|c|c|c|c|}
\hline Characteristics & Total, $\mathrm{n}=146$ & Control Arm 1, $\mathrm{n}=46$ & Tapering Arm 2, $\mathrm{n}=47$ & Stopping Arm 3, $\mathrm{n}=53$ & $\mathrm{p}$ \\
\hline Age, yrs & 56.12 & 55 & 56.77 & 56.51 & 0.773 \\
\hline Female & $56.8(83)$ & $52.2(24)$ & $57.4(27)$ & $60.4(32)$ & 0.710 \\
\hline Remission duration, mos & 18.9 & 18.2 & 18.6 & 22.6 & 0.362 \\
\hline DAS28 & 1.71 & 1.66 & 1.61 & 1.85 & 0.136 \\
\hline RAID & 1.19 & 1.19 & 0.96 & 1.36 & 0.529 \\
\hline Glucocorticoid use & $23.3(34)$ & $26.1(12)$ & $23.4(11)$ & $20.8(11)$ & 0.822 \\
\hline Other cDMARD use ${ }^{* *}$ & $13.7(20)$ & $13.0(6)$ & $12.8(6)$ & $15.1(8)$ & 0.933 \\
\hline RF-positive & $56.2(82)$ & $45.7(21)$ & $66.0(31)$ & $56.6(30)$ & 0.142 \\
\hline ACPA-positive & $56.2(82)$ & $56.5(26)$ & $57.4(27)$ & $54.7(29)$ & 0.961 \\
\hline MBDA-positive & $43.2(63)$ & $34.8(16)$ & $34.0(16)$ & $58.5(31)$ & 0.018 \\
\hline
\end{tabular}

Data are given as means or \% (n). Values in bold face are statistically significant. Tumor necrosis factor inhibitors: * tocilizumab, abatacept; ** leflunomide, sulfasalazine, hydroxychloroquine. DAS28: 28-joint count Disease Activity Score (using erythrocyte sedimentation rate); RAID: Rheumatoid Arthritis Impact of Disease questionnaire; bDMARD: biological disease-modifying antirheumatic drugs; cDMARD: conventional DMARD; RF: rheumatoid factor; ACPA: anticitrullinated protein antibodies; MBDA: multibiomarker disease activity.

distribution of treatment costs and their reduction in the 3 study arms in relation to risk predictors. Direct treatment costs for double-negative and single-positive groups $(n=41)$ would have been $€ 372,245.16$ for full-dose treatment over 1 year. Tapering and stopping DMARD in these low-risk relapse groups allowed a reduction of $€ 219,712.03$ in drug costs, which leads to an average cost reduction of $€ 5358.83$ per patient. There was no difference in treatment costs using ACPA or MBDA as first-order risk factors. When using only ACPA status for relapse prediction, overall cost reduction was lower with $€ 184,580.00$ and an average cost reduction of $€ 4394.76$ per patient with an overall higher relapse risk.

\section{DISCUSSION}

Achieving sustained remission with DMARD treatment has become a realistic goal in the treatment of RA. Several studies have addressed DMARD tapering in patients with RA in sustained DAS28 remission and low disease activity $4,15,16,17$ and DMARD tapering has also been implemented in EULAR and ACR guidelines ${ }^{18,19}$. While it is clear that DMARD tapering is only feasible in patients with completely absent or very low signs and symptoms of disease $^{4}$, the question arises as to which patients can successfully taper or even stop DMARD treatment. The role of markers that increase or decrease the likelihood for disease relapse in patients tapering DMARD is of interest, because only some patients with RA can maintain remission after tapering the drugs. ACPA and MBDA are of interest in this respect, because ACPA status has no relation and MBDA only limited relation to clinical disease activity in $\mathrm{RA}^{20}$. Hence, some patients with RA in stable remission are characterized by positive ACPA and/or signs of biochemical disease activity reflected by moderate to high MBDA scores. Our previous data revealed that positive ACPA status and moderate/high MBDA scores predict the relapse risk in patients tapering DMARD ${ }^{7}$.

In addition to the identification of patients able to taper treatment, economic considerations also come into focus $12,22,23,24$. This is largely because bDMARD costs are high, and successful tapering and stopping of these drugs in patients with RA in remission leads to a substantial reduction in DMARD costs. Importantly, drug costs have been shown to affect the treatment decisions of rheumatologists ${ }^{5}$. Today, with more effective and accessible DMARD to treat RA, potential overtreatment of patients with RA in remission may deserve recognition equal to that given to potential undertreatment of patients not in remission. Barnabe and colleagues stated that sustained remission can lead to decreased healthcare service use ${ }^{25}$. Michaud and colleagues analyzed the outcome and costs of using MBDA testing to improve the assessment of disease activity and subsequent changes in treatment decisions and showed that using MBDA score can reduce costs in patients with RA as well as improve their functional status ${ }^{26}$.

Our present study shows that tapering or stopping DMARD achieves savings of direct healthcare costs, if the likelihood for relapse is low and the patients can permanently stay on a tapered DMARD regimen or could even stop treatment. RA patients with a low risk for relapse are at risk for overtreatment, while in those with high risk for relapse, tapering or even stopping DMARD treatment is not a feasible option. Our data show that overtreatment can be prevented and costs can be saved if tapering is performed in RA patients with low relapse risk based on ACPA and MBDA status. Such an approach can combine the interests of patients (safety, overtreatment), rheumatologists (personalized medicine), and health insurance (costs). For obvious reasons, prediction of relapses based on MBDA and ACPA cannot be $100 \%$ correct

Personal non-commercial use only. The Journal of Rheumatology Copyright @ 2019 . All rights reserved. 


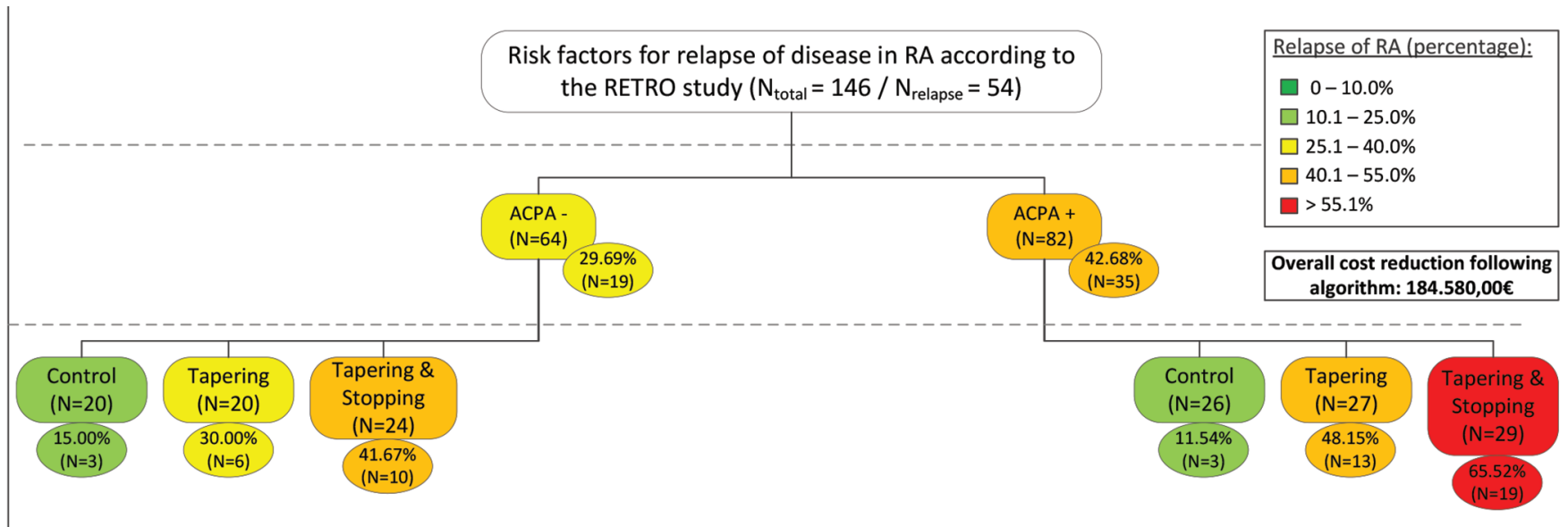

Figure 2. Relapse risk stratification using ACPA status only (Model 1). Green $=$ low relapse risk $(<25 \%$ over $1 \mathrm{yr})$; yellow/orange $=$ moderate relapse risk $(26-$ $55 \%$ over $1 \mathrm{yr})$; red $=$ high relapse risk $(>55 \%$ over $1 \mathrm{yr})$. ACPA: anticitrullinated protein antibodies.

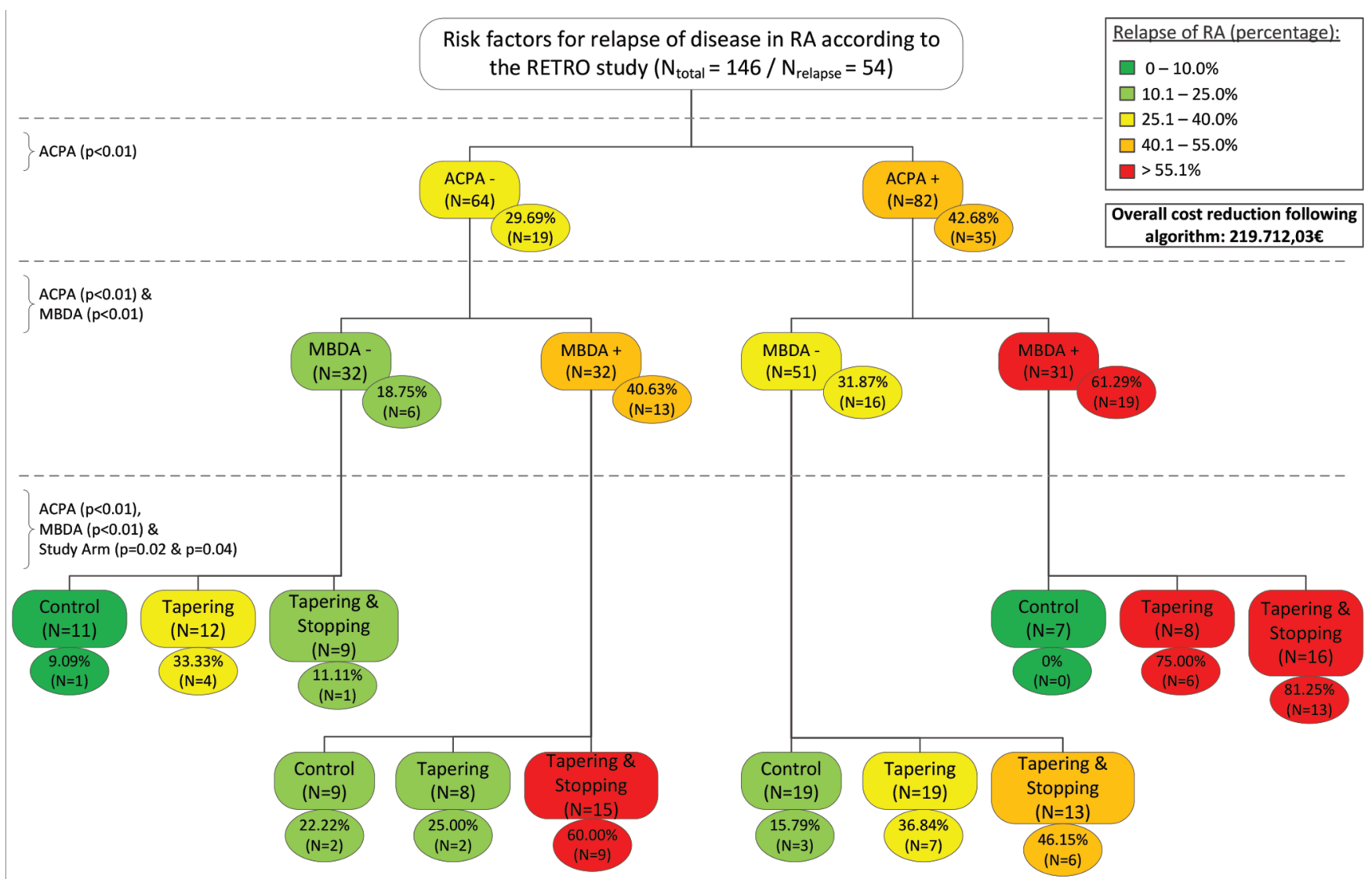

Figure 3. Relapse risk stratification using ACPA status combined with MBDA score (Model 2). Green = low relapse risk $(<25 \%$ over $1 \mathrm{yr})$; yellow/orange $=$ moderate relapse risk (26-55\% over $1 \mathrm{yr})$; red = high relapse risk ( $>55 \%$ over $1 \mathrm{yr})$. RA: rheumatoid arthritis; ACPA: anticitrullinated protein antibodies; MBDA: multibiomarker disease activity.

on the individual patient's level. Hence, it has to be taken into account that even the combination of negative ACPA and low MBDA is no absolute guarantee against relapse, and the combination of positive ACPA and moderate/high MBDA is no absolute prediction of relapse.
It should be mentioned that cost reductions in DMARD reported in our study refer to prices of DMARD in Germany. Hence, the absolute numbers of DMARD savings may vary from country to country based on actual drug costs, the frequency of biosimilars used, and the presence 
Table 2A. Cost reduction adapted to risk factors for relapse: model based on ACPA status only.

\begin{tabular}{|c|c|c|c|}
\hline Arms & ACPA- & $\mathrm{ACPA}+$ & Total \\
\hline Arm 1 (Control) & $\begin{array}{c}\mathrm{BL}=€ 128,302.32 \\
\mathrm{M} 12=€ 128,302.51 \\
-€ 0^{*} \\
(\mathrm{n}=17)\end{array}$ & $\begin{array}{c}\mathrm{BL}=€ 209,984.76 \\
\mathrm{M} 12=€ 209,984.76 \\
-€ 0^{*} \\
(\mathrm{n}=23)\end{array}$ & $\begin{array}{c}\mathrm{BL}=€ 338,287.08 \\
\mathrm{M} 12=€ 338,287.27 \\
-€ 0^{*} \\
(\mathrm{n}=40)^{*}\end{array}$ \\
\hline Arm 2 (Tapering) & $\begin{array}{c}\mathrm{BL}=€ 92,962.44 \\
\mathrm{M} 12=€ 44,436.58 \\
-€ \mathbf{4 8 , 5 2 5 . 8 6} \\
(\mathrm{n}=14)\end{array}$ & $\begin{array}{c}\mathrm{BL}=€ 222,054.56 \\
\mathrm{M} 12=€ 114,116.31 \\
-€ \mathbf{1 0 7 , 9 3 8 . 2 5} \\
(\mathrm{n}=14)\end{array}$ & $\begin{array}{c}\mathrm{BL}=€ 315,017.00 \\
\mathrm{M} 12=€ 158,552.89 \\
-€ \mathbf{1 5 6 , 4 6 4 . 1 1} \\
(\mathrm{n}=28)\end{array}$ \\
\hline Arm 3 (Stopping) & $\begin{array}{c}\mathrm{BL}=€ 39,585.12 \\
\mathrm{M} 12=€ 11,469.26 \\
-€ \mathbf{2 8 , 1 1 5 . 8 6} \\
(\mathrm{n}=14)\end{array}$ & $\begin{array}{c}\mathrm{BL}=€ 49,231.20 \\
\mathrm{M} 12=€ 13,883.65 \\
-€ 35,347.55^{* *} \\
(\mathrm{n}=10)\end{array}$ & $\begin{array}{c}\mathrm{BL}=€ 39,585.12 \\
\mathrm{M} 12=€ 11,469.26 \\
-€ \mathbf{2 8 , 1 1 5 . 8 6} \\
(\mathrm{n}=14)\end{array}$ \\
\hline All & & & $\begin{array}{c}\mathrm{BL}=€ 354,602.12 \\
\mathrm{M} 12=€ 170,022.12 \\
-€ \mathbf{1 8 4 , 5 8 0 . 0 0} \\
(\mathrm{n}=42)\end{array}$ \\
\hline
\end{tabular}

Table $2 B$. Cost reduction adapted to risk factors for relapse: model based on ACPA status combined with MBDA score.

\begin{tabular}{|c|c|c|c|c|c|}
\hline Arms & ACPA-/MBDA- & ACPA-/MBDA+ & $\mathrm{ACPA}+\mathrm{MBDA}-$ & $\mathrm{MBDA}+/ \mathrm{ACPA}+$ & Total \\
\hline Arm 1 (Control) & $\begin{array}{c}\mathrm{BL}=€ 80,286.80 \\
\mathrm{M} 12=€ 80,286.80 \\
-€ 0 * \\
(\mathrm{n}=10)\end{array}$ & $\begin{aligned} \mathrm{BL}= & € 64,853.52 \\
\mathrm{M} 12= & € 64,853.52 \\
& -€ 0^{*} \\
& (\mathrm{n}=7)\end{aligned}$ & $\begin{array}{c}\mathrm{BL}=€ 159,185.88 \\
\mathrm{M} 12=€ 159,185.88 \\
-€ 0 * \\
(\mathrm{n}=16)\end{array}$ & $\begin{array}{c}\mathrm{BL}=€ 73,568.88 \\
\mathrm{M} 12=€ 73,568.88 \\
-€ 0^{*} \\
(\mathrm{n}=7)\end{array}$ & $\begin{array}{c}\mathrm{BL}=€ 377,895.08 \\
\mathrm{M} 12=€ 377,895.08 \\
-€ 0^{*} \\
(\mathrm{n}=40)\end{array}$ \\
\hline Arm 2 (Tapering) & $\begin{array}{c}\mathrm{BL}=€ 70,839.24 \\
\mathrm{M} 12=€ 27,619.32 \\
-€ \mathbf{4 3 , 2 1 9 . 9 2} \\
(\mathrm{n}=8)\end{array}$ & $\begin{array}{c}\mathrm{BL}=€ 35,983.20 \\
\mathrm{M} 12=€ 16,817.20 \\
-€ \mathbf{1 9 , 1 6 6 . 0 0} \\
(\mathrm{n}=6)\end{array}$ & $\begin{array}{c}\mathrm{BL}=€ 189,480.00 \\
\mathrm{M} 12=€ 91,013.56 \\
-€ \mathbf{9 8 , 4 6 6 . 4 4} \\
(\mathrm{n}=12)\end{array}$ & $\begin{array}{c}\mathrm{BL}=€ 46,434.56 \\
\mathrm{M} 12=€ 23,102.75 \\
-€ 23,331.81^{* *} \\
(\mathrm{n}=2)\end{array}$ & $\begin{array}{c}\mathrm{BL}=€ 296,302.44 \\
\mathrm{M} 12=€ 135,450.08 \\
-€ \mathbf{1 6 0 , 8 5 2 . 3 6} \\
(\mathrm{n}=26)\end{array}$ \\
\hline Arm 3 (Stopping) & $\begin{array}{c}\mathrm{BL}=€ 42,208.32 \\
\mathrm{M} 12=€ 9446.40 \\
-€ \mathbf{3 2 , 7 6 1 . 9 2} \\
(\mathrm{n}=8)\end{array}$ & $\begin{array}{c}\mathrm{BL}=€ 11,236.80 \\
\mathrm{M} 12=€ 1499.06 \\
-€ 9737.74 * * \\
(\mathrm{n}=6)\end{array}$ & $\begin{array}{c}\mathrm{BL}=€ 33,734.40 \\
\mathrm{M} 12=€ 7636.65 \\
-€ \mathbf{2 6 , 0 9 7 . 7 6} \\
(\mathrm{n}=7)\end{array}$ & $\begin{array}{c}\mathrm{BL}=€ 24,406.80 \\
\mathrm{M} 12=€ 6247.00 \\
-€ 18,159.80 * * \\
(\mathrm{n}=3)\end{array}$ & $\begin{array}{c}\mathrm{BL}=€ 75,942.72 \\
\mathrm{M} 12=€ 17,083.05 \\
-€ \mathbf{5 8 , 8 5 9 . 6 7} \\
(\mathrm{n}=15)\end{array}$ \\
\hline All & & & & & $\begin{array}{c}\mathrm{BL}=€ 372,245.16 \\
\mathrm{M} 12=€ 152,533.13 \\
-€ \mathbf{2 1 9 , 7 1 2 . 0 3} \\
(\mathrm{n}=41)\end{array}$ \\
\hline
\end{tabular}

Values in bold face are absolute saved costs. * No cost reduction, owing to study design. ** No cost reduction, owing to high relapse risk. BL: baseline assessment at start of tapering; M12: Month 12 followup; ACPA: anticitrullinated protein antibodies; MBDA: multibiomarker disease activity.

of insurance or pharmacy contracts regulating DMARD prices. Nonetheless, the concept that tapering and stopping DMARD in a low-relapse risk population is cost-effective seems to be generalizable. Further, it needs to be mentioned that data are based on a small patient cohort, and new MBDA cutoffs adjusted for age and weight have not been taken into account ${ }^{27}$. Another limitation of our study is that indirect costs were not analyzed; they can sometimes be substantially higher than direct $\operatorname{costs}^{28}$. Further, we analyzed patients in DAS28 remission, which at first sight contrasts with the ACR guideline stating that patients' medication should not be tapered unless the patient is in ACR/EULAR remission ${ }^{19}$. Our data suggest that tapering can be successful in a fraction of patients who are in DAS28-ESR remission. Also, when analyzing the subset of patients fulfilling the Boolean remission criteria at entry, MBDA remained as an independent predictor for relapse $(\mathrm{p}=0.01)$. However, in the subset of patients fulfilling SDAI remission at entry, MBDA lost its independent prediction for relapse $(\mathrm{p}=0.24)$, which is most likely because numerical CRP values are included in both Simplified Disease Activity Index (SDAI) and MBDA scores, and thus a smaller number of patients show elevated MBDA scores in SDAI remission.

Our study presents an approach toward cost-effective stratified DMARD tapering. It addresses the current challenges in handling stable remission in patients with RA, the possibility of a biomarker-based stratified DMARD tapering approach, and the reduction in DMARD costs resulting from such a concept. 


\section{ACKNOWLEDGMENT}

The present work was performed in fulfillment (by co-author Melanie Hagen) of the requirements for obtaining the degree "Dr. med." at the Friedrich-Alexander-University Erlangen-Nuremberg.

\section{REFERENCES}

1. McInnes IB, Schett G. The pathogenesis of rheumatoid arthritis. N Engl J Med 2011;365:2205-19.

2. Smolen JS, Aletaha D, Bijlsma JW, Breedveld FC, Boumpas D, Burmester G, et al; T2T Expert Committee. Treating rheumatoid arthritis to target: recommendations of an international task force. Ann Rheum Dis 2010;69:631-7.

3. Aga AB, Lie E, Uhlig T, Olsen IC, Wierod A, Kalstad S, et al. Time trends in disease activity, response and remission rates in rheumatoid arthritis during the past decade: results from the NOR-DMARD study 2000-2010. Ann Rheum Dis 2015;74:381-8.

4. Schett G, Emery P, Tanaka Y, Burmester G, Pisetsky DS, Naredo E, et al. Tapering biologic and conventional DMARD therapy in rheumatoid arthritis: current evidence and future directions. Ann Rheum Dis 2016;75:1428-37.

5. Hifinger M, Hiligsmann M, Ramiro S, Watson V, Severens JL, Fautrel B, et al. Economic considerations and patients' preferences affect treatment selection for patients with rheumatoid arthritis: a discrete choice experiment among European rheumatologists. Ann Rheum Dis 2017;76:126-32.

6. Haschka J, Englbrecht M, Hueber AJ, Manger B, Kleyer A, Reiser $\mathrm{M}$, et al. Relapse rates in patients with rheumatoid arthritis in stable remission tapering or stopping antirheumatic therapy: interim results from the prospective randomised controlled RETRO study. Ann Rheum Dis 2016;75:45-51.

7. Rech J, Hueber AJ, Finzel S, Englbrecht M, Haschka J, Manger B, et al. Prediction of disease relapses by multibiomarker disease activity and autoantibody status in patients with rheumatoid arthritis on tapering DMARD treatment. Ann Rheum Dis 2016;75:1637-44.

8. Aletaha D, Neogi T, Silman AJ, Funovits J, Felson DT, Bingham CO 3rd, et al. 2010 Rheumatoid arthritis classification criteria: an American College of Rheumatology/European League Against Rheumatism collaborative initiative. Arthritis Rheum 2010; 62:2569-81.

9. Prevoo ML, van 't Hof MA, Kuper HH, van Leeuwen MA, van de Putte LB, van Riel PL. Modified disease activity scores that include twenty-eight-joint counts. Development and validation in a prospective longitudinal study of patients with rheumatoid arthritis. Arthritis Rheum 1995;38:44-8.

10. Centola M, Cavet G, Shen Y, Ramanujan S, Knowlton N, Swan KA, et al. Development of a multi-biomarker disease activity test for rheumatoid arthritis. PLoS One 2013;8:e60635.

11. Curtis JR, van der Helm-van Mil AH, Knevel R, Huizinga TW, Haney DJ, Shen Y, et al. Validation of a novel multibiomarker test to assess rheumatoid arthritis disease activity. Arthritis Care Res 2012;64:1794-803

12. Hirata S, Dirven L, Shen Y, Centola M, Cavet G, Lems WF, et al. A multi-biomarker score measures rheumatoid arthritis disease activity in the BeSt study. Rheumatology 2013;52:1202-7.

13. Li W, Sasso EH, Emerling D, Cavet G, Ford K. Impact of a multi-biomarker disease activity test on rheumatoid arthritis treatment decisions and therapy use. Curr Med Res Opin 2013;29:85-92.

14. Hirata S, Li W, Kubo S, Fukuyo S, Mizuno Y, Hanami K, et al. Association of the multi-biomarker disease activity score with joint destruction in patients with rheumatoid arthritis receiving tumor necrosis factor-alpha inhibitor treatment in clinical practice. Mod Rheumatol 2016;26:850-6.

15. Tanaka Y, Hirata S, Kubo S, Fukuyo S, Hanami K, Sawamukai N, et al. Discontinuation of adalimumab after achieving remission in patients with established rheumatoid arthritis: 1-year outcome of the HONOR study. Ann Rheum Dis 2015;74:389-95.

16. van der Woude D, Young A, Jayakumar K, Mertens BJ, Toes RE, van der Heijde D, et al. Prevalence of and predictive factors for sustained disease-modifying antirheumatic drug-free remission in rheumatoid arthritis: results from two large early arthritis cohorts. Arthritis Rheum 2009;60:2262-71.

17. Klarenbeek NB, van der Kooij SM, Guler-Yuksel M, van Groenendael JH, Han KH, Kerstens PJ, et al. Discontinuing treatment in patients with rheumatoid arthritis in sustained clinical remission: exploratory analyses from the BeSt study. Ann Rheum Dis 2011;70:315-9.

18. Smolen JS, Landewe R, Breedveld FC, Buch M, Burmester G, Dougados M, et al. EULAR recommendations for the management of rheumatoid arthritis with synthetic and biological disease-modifying antirheumatic drugs: 2013 update. Ann Rheum Dis 2014;73:492-509.

19. Singh JA, Saag KG, Bridges SL Jr, Akl EA, Bannuru RR, Sullivan MC, et al. 2015 American College of Rheumatology guideline for the treatment of rheumatoid arthritis. Arthritis Rheumatol 2016;68:1-26.

20. Fleischmann R, Connolly SE, Maldonado MA, Schiff M. Brief report: Estimating disease activity using multi-biomarker disease activity scores in rheumatoid arthritis patients treated with abatacept or adalimumab. Arthritis Rheumatol 2016;68:2083-9.

21. Schoels M, Wong J, Scott DL, Zink A, Richards P, Landewe R, et al. Economic aspects of treatment options in rheumatoid arthritis: a systematic literature review informing the EULAR recommendations for the management of rheumatoid arthritis. Ann Rheum Dis 2010;69:995-1003.

22. van der Velde G, Pham B, Machado M, Ieraci L, Witteman W, Bombardier C, et al. Cost-effectiveness of biologic response modifiers compared to disease-modifying antirheumatic drugs for rheumatoid arthritis: a systematic review. Arthritis Care Res 2011;63:65-78.

23. Joensuu JT, Huoponen S, Aaltonen KJ, Konttinen YT, Nordstrom D, Blom M. The cost-effectiveness of biologics for the treatment of rheumatoid arthritis: a systematic review. PLoS One 2015;10:e0119683.

24. Kalkan A, Roback K, Hallert E, Carlsson P. Factors influencing rheumatologists' prescription of biological treatment in rheumatoid arthritis: an interview study. Implement Sci 2014;9:153.

25. Barnabe C, Thanh NX, Ohinmaa A, Homik J, Barr SG, Martin L, et al. Healthcare service utilisation costs are reduced when rheumatoid arthritis patients achieve sustained remission. Ann Rheum Dis 2013;72:1664-8.

26. Michaud K, Strand V, Shadick NA, Degtiar I, Ford K, Michalopoulos SN, et al. Outcomes and costs of incorporating a multibiomarker disease activity test in the management of patients with rheumatoid arthritis. Rheumatology 2015;54:1640-9.

27. Curtis J, Greenberg JD, Harrold LR, Kremer JM, Lynn Palmer JL. Influence of obesity, age, and comorbidities on the multi-biomarker disease activity test in rheumatoid arthritis. Semin Arthritis Rheum 2018;47:472-7.

28. Pugner KM, Scott DI, Holmes JW, Hieke K. The costs of rheumatoid arthritis: an international long-term view. Semin Arthritis Rheum 2000;29:305-20. 